\title{
The Simulation, Design and Implementation of Bandpass Filters in Rectangular Waveguides
}

\author{
Sarun Choocadee, Somsak Akatimagool* \\ Department of Teaching Training in Electrical Engineering, Faculty of Technical Education, \\ King Mongkut's University of Technology North Bangkok, Thailand
}

\begin{abstract}
This paper presents the simulation, design and implementation of bandpass filters in rectangular waveguides. The filters are simulated and designed by using a numerical analysis program based on the Wave Iterative Method (WIM) called WFD (Waveguide Filter Design) simulation. This simulation program can analyze the electrical network of filters. The sample waveguide filter is designed based on a proposed structure consisting of three circuits serving as a simple bandpass filter with two inductive irises and three inductive irises in a cascade. We determined that the operating frequency was equal to approximately 4.20-4.84 GHz. The waveguide filters were then implemented using an aluminum material. The analyzed results for the inductive iris obtained with the WFD program agree well with the results of CST Microwave Studioß. Finally, the measured results of sample waveguide filters are consistent with the simulation tool.
\end{abstract}

Keywords Inductive Irises, Simulation Program, Bandpass Filter, Waveguide Filters Design, Wave Iterative Method

\section{Introduction}

Presently, the development of technologies for wireless communications, particularly for use in the microwave frequency range, is critical to developing the economy and domestic industry and contributes to continued technology research and development. Microwaves filters were found to be necessary because a communication system can be used to separate the signals that would be needed for use. In several articles, researchers have studied the filters in waveguides, and the results of simulation programs are most interesting[1-4].

The most popular waveguide filter is an iris filter[5-8]. The researchers cited on 3D simulators, such as CST Microwave Studio $^{\circledR}$ and Advanced Design System (ADS), to design iris waveguide filters. The problems with the design of filters often include a lack of equipment and tools used to design and build them. Numerical methods related to electromagnetic waves have caused a revolution in microwave engineering; techniques such as FDTD (Finite Differential Time Domain)[1], Smoothed Piecewise Linear model (SPEL)[2], TLM (Transmission Line Matrix)[3], and the Moment method[4] have been developed. Each method has respective disadvantages and limitations for use in research and education. Some analysis methods for designing waveguide circuits are available in[1] to[8].

* Corresponding author:

ssa@kmutnb.ac.th (S.Akatimagool)

Published online at http://journal.sapub.org/eee

Copyright (C) 2012 Scientific \& Academic Publishing. All Rights Reserved
Recently reported waveguide filter has several structures. Many accurate techniques have also been proposed but have complex algorithms[9-12]. The Ref.[9] explains a new method based on inverse scattering theory of single-mode planar waveguides in order to obtain a desired TE-mode profile. However, this method can also be expanded to analyze TM modes and for designing multi-mode planar waveguides. Also, an accurate CAD tool based on the merging the prototype synthesis and the structure extraction has been presented in[11] for the particular case of inline inductive waveguide filters.

We observed that the most inductive iris in a waveguide has been designed with high accuracy through approximate modeling utilizing the full-wave analysis method. Therefore, the development and optimization of methods are necessary and important for an efficient electromagnetic waveguide analysis simulation tool.

This paper proposes a simulation, design and implementation of bandpass filters by using the novel technique of Wave Iterative Method (WIM)[12], the CST Microwave Studio $^{\circledR}$ and a network analyzer. The result can be used in microwave filter design for satellite communications and applied in education and in the telecommunication industry.

\section{Numerical Analysis}

An analysis of the electromagnetic wave properties within a waveguide consists of TE and TM field components. Most wave analyses will be calculated in the spectral domain based on the series integration equation to present the electromagnetic field and to analyze the two-port network pa- 
rameters such that the result of the frequency response reflects the characteristic of various filter circuits. In this research, we will present the cooperation of waves between the spatial domain and the frequency domain.

The calculating concept for electromagnetic wave propagation in a waveguide is based on the Wave Iterative Method (WIM). The operating process, shown in Figure 1, will present the amplitude and direction of the incident, reflected, and transmitted waves that propagate in the waveguide iris. On the iris, the waves are calculated in the spatial domain (Real) and the waves in free space are calculated in the frequency domain (Modes). To alternate between both domains, we use the Fast Fourier Transform $(F F T)$ to reduce the computation time.

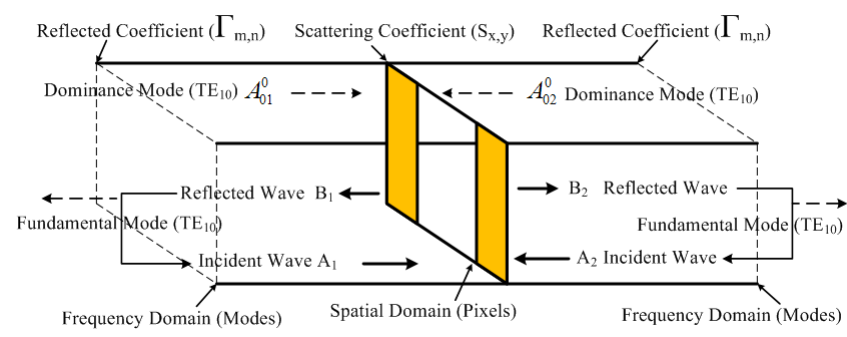

Figure 1. Wave Iterative Method in a rectangular waveguide

In Figure 1, the first step, the $T E_{10}$ field, which is the dominant mode, will propagate into the iris circuit, and then the higher-mode waves caused by the scatter on the conductor surface will be incident and will reflect within the waveguide. An infinite numbers of reflected and transmitted waves are produced at the iris circuit interface. Finally, the calculation of waves will use the principle of mode matching at the input and output ports of the waveguide. The basic functions of TE field components in the electric wall for an inductive obstacle are

$$
\begin{aligned}
& \vec{E}_{x(x, y)}^{\alpha}=\mathrm{K}_{\mathrm{x}}^{\alpha} \cos \left(\frac{m \pi x}{a}\right) \sin \left(\frac{n \pi y}{b}\right), \\
& \vec{E}_{y(x, y)}^{\alpha}=-\mathrm{K}_{\mathrm{y}}^{\alpha} \sin \left(\frac{m \pi x}{a}\right) \cos \left(\frac{n \pi y}{b}\right),
\end{aligned}
$$

where $\alpha$ refers to the TE mode and $K$ is the value constant with respect to the $x$ and $y$ directions. Thus, the initial value of the $T E_{10}$ dominant wave $\left(A_{x, y}\right)$ in the spatial domain is

$$
A_{x, y}^{(0)}=-\sqrt{\frac{2}{a b}} \sin \left(\frac{\pi x}{a}\right),
$$

where $a$ and $b$ are the dimensions of the waveguide. The reflected waves $\left(B_{x, y}\right)$ in $n$ iterations on the iris circuit are

$$
B_{x, y}^{(n)}=S_{x, y} A_{x, y}^{(n-1)} .
$$

The scattering parameter $\left(S_{x, y}\right)$ of waves in the spatial domain of a two-port network is defined as

$$
\left[S_{x, y}\right]=\left[\begin{array}{cc}
S_{11(x, y)} & S_{12(x, y)} \\
S_{21(x, y)} & S_{22(x, y)}
\end{array}\right],
$$

where $S_{11}$ and $S_{22}$ are equal to -1 in the good conductor area and $S_{12}$ and $S_{21}$ are equal to +1 for the free space. To transform the wave from the spatial domain to the spectrum do- main, we use the Fast Fourier Transform (FFT)

$$
B_{m, n}^{(n)}=F F T\left(B_{x, y}^{(n)}\right) .
$$

At the input port of the waveguide, the reflected waves $\left(B_{m, n}\right)$ of higher-order modes will feed back into the obstacle of the waveguide. The equation for incident waves $\left(A_{m, n}\right)$ is

$$
A_{m, n}^{(n)}=\Gamma_{m, n} B_{m, n}^{(n)}+A_{1,0}^{T E} / T M
$$

where $\left(A_{1,0}\right)$ is an initial exciting source. The reflection coefficient $\left(\Gamma_{m, n}\right)$ of waves in the spectrum domain at the input and output ports of a rectangular waveguide can be written as

$$
\Gamma_{m, n}=\Gamma_{m, n}^{T E / T M}=\frac{1-Z_{0} Y_{m, n}^{T E} / T M}{1+Z_{0} Y_{m, n}^{T E} / T M},
$$

where $Z_{0}$ is the intrinsic impedance of the dominant mode and $Y_{m, n}$ is the TE/TM admittance modes with orders of $m$ and $n$, which can be expressed as

$$
\begin{gathered}
Y_{m, n}^{T E}=\frac{1}{j \omega \mu}\left[\left(\frac{m \pi}{a}\right)^{2}+\left(\frac{n \pi}{b}\right)^{2}-\omega^{2} \mu \varepsilon\right], \\
\text { and } Y_{m, n}^{T M}=\frac{j \omega \varepsilon}{\left(\frac{m \pi}{a}\right)^{2}+\left(\frac{n \pi}{b}\right)^{2}-\omega^{2} \mu \varepsilon} .
\end{gathered}
$$

Therefore, considering the waves on the iris, the waves $\left(A_{m, n}\right)$ will be transformed by using the Inverse Fast Fourier Transform (IFFT) to analyze the basic functions of TE/TM field components to come back to the spatial domain as

$$
A_{x, y}^{(n)}=\text { Pixel_IFFT }\left(B_{m, n}^{(n)}\right) .
$$

Therefore, the relation of incident waves at $n$ iterations in the calculating process is

$$
A_{i}^{(n)}=(\Gamma S)^{(n-1)} A_{i}^{(n-1)}+A_{0 i},
$$

where $A$ is the incident wave, $n$ is the number of iterations, $i$ is the number of waveguide ports, $\Gamma$ is the reflection coefficient at the input/output port and $S$ is the scattering parameter of the iris circuit.

In considering the convergence condition, the incident waves at $n$ iterations on the iris area tend toward zero. We can obtain the input reflection coefficient of the iris circuit of the dominant mode $\left(T E_{10}\right)$ as

$$
\Gamma_{i n(1,0)}=\frac{B_{1,0}^{T E} / T M}{A_{1,0}^{T E / T M}}=\frac{\sum_{n=1}^{N} B(n)_{1,0}^{T E / T M}}{A_{1,0}^{T E} / / M} .
$$

The input impedance equation of an iris can be written as

$$
Z_{i n(1,0)}=Z_{0}\left(\frac{\Gamma_{i n(1,0)}-1}{2 \Gamma_{i n(1,0)}}\right) .
$$

\section{WFD Simulation Results}

The simulation program conducts a numerical analysis using the Wave Iterative Method. This simulation program, called WFD (Waveguide Filter Design), consists of a main menu, parameter setup, and a design and display window, as 
shown in Figure 2. The WFD is constructed by using the GUI (Graphic User Interface) of MATLAB ${ }^{\circledR}$. The user can set up the initial values that are used for calculating the two ports of the network, calculate the properties of the inductive iris and select the display windows of the simulated results. With the simulation program, it is possible to analyze and design a waveguide filter.

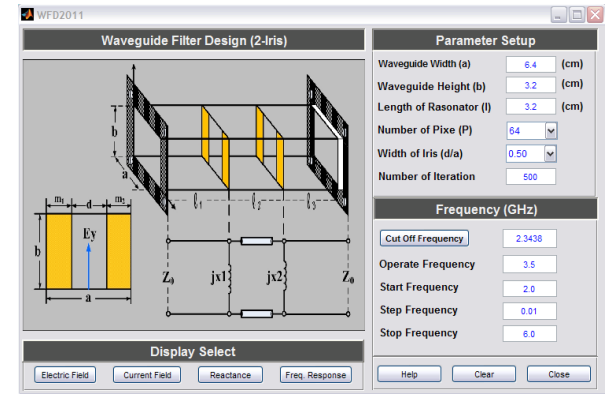

(a) Menu window

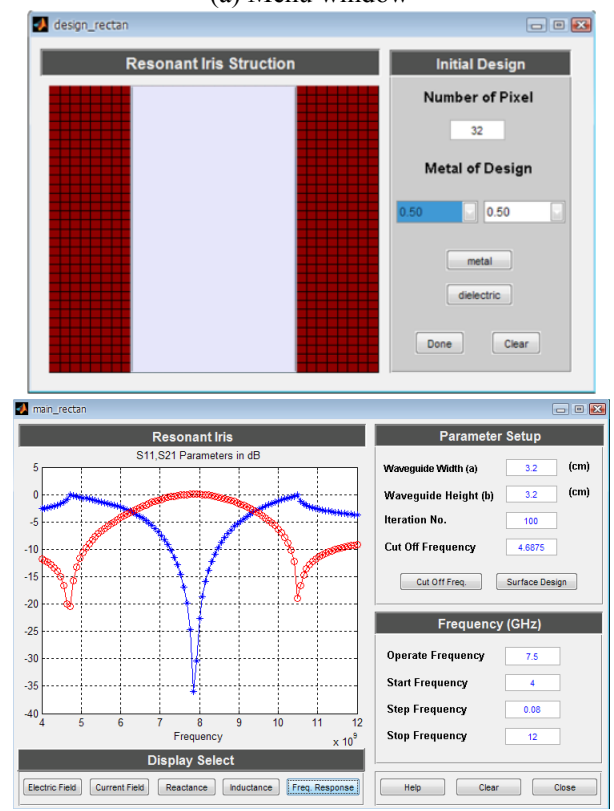

(b) Parameter setup and design and display windows Figure 2. Waveguide Filter Design (WFD) Simulation
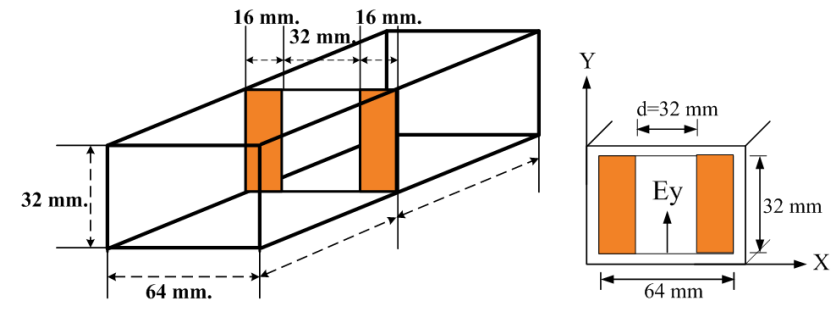

Figure 3. Inductive iris structure

We present an inductive iris analysis in a rectangular waveguide obtained using the developed WFD simulation program. The WFD simulated result will be compared with the result obtained from CST Microwave Studio ${ }^{\circledR}$ software. The desired frequency response characteristic of the high-pass filter (HPF) was considered. The dimensions of the rectangular waveguide consist of a width (a) equal to 64 $\mathrm{mm}$, a height (b) equal to $32 \mathrm{~mm}$ and the iris width (d) equal to $32 \mathrm{~mm}$, as shown in Figure 3. The cutoff frequency of the waveguide is $2.34 \mathrm{GHz}$.

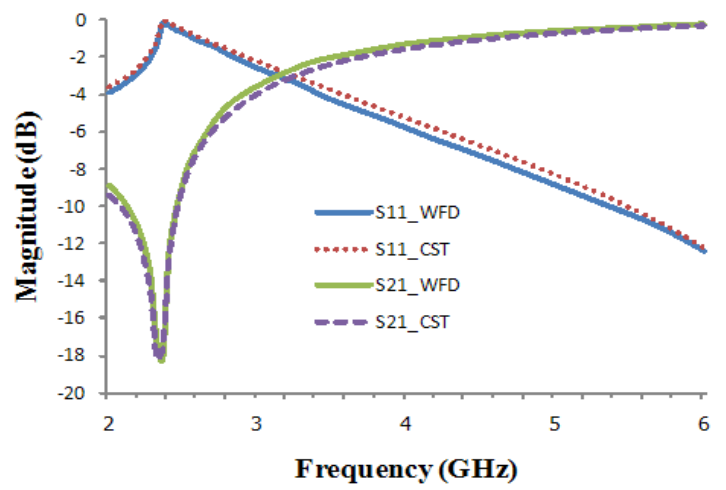

Figure 4. Comparison of $\mathrm{dB}(\mathrm{S} 11)$ and $\mathrm{dB}(\mathrm{S} 21)$ of the high-pass filter between the WFD and CST tools at $d / a=0.5$

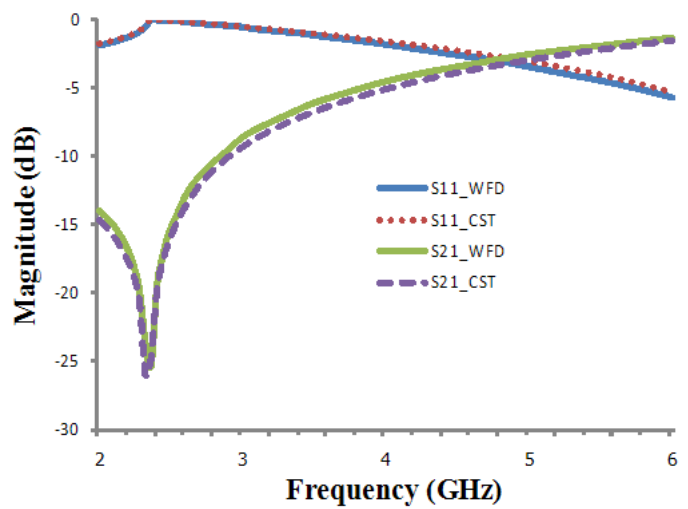

Figure 5. Comparison of $\mathrm{dB}(\mathrm{S} 11)$ and $\mathrm{dB}(\mathrm{S} 21)$ of the high-pass filter between the WFD and CST tools at $d / a=0.375$

Figure 4 presents a comparison of $\mathrm{dB}(\mathrm{S} 11)$ and $\mathrm{dB}(\mathrm{S} 21)$ of the inductive iris circuit obtained with the WFD tool and CST simulation. The width of the iris (d) is equal to $32 \mathrm{~mm}$, we can obtain the $-3 \mathrm{~dB}$ cutoff frequency at $3.43 \mathrm{GHz}$. This comparison revealed good agreement. The error of the simulated results is less than $\pm 2 \%$.

Figure 5 presents the comparison of $\mathrm{dB}(\mathrm{S} 11)$ and $\mathrm{dB}(\mathrm{S} 21)$ of the inductive iris between the WFD and CST simulations for an inductive iris circuit width equal to $40 \mathrm{~mm}$. We can obtain the $-3 \mathrm{~dB}$ cutoff frequency at $4.82 \mathrm{GHz}$. This comparison revealed good agreement. The error of the simulated results is less than $\pm 2.5 \%$. Thus, the WFD simulation is an efficient simulation tool.

\section{Design and Implementation of Filters}

\subsection{Bandpass Filter Design}

In this paper, we present the inductive irises in a rectangular metal waveguide by using the simulation program. The usable conductor of irises is a good conductor. The main specifications of the sample bandpass filter for a C-band multiplexer are shown in Figure 6. The bandpass waveguide filter consists of 3 configurations, one iris, two irises and three inductive irises placed in a rectangular waveguide, and 
is designed by using the formulas of a low-pass Chebyshev prototype filter. The width $(a)$ and height $(b)$ of the waveguide are 64 and $32 \mathrm{~mm}$, respectively. The element values of Chebyshev filters have a ripple $=0.1 \mathrm{~dB}$ with $50 \Omega$ of system impedance. We will calculate and optimize each filter parameter to cover the frequency range of $4.20-4.84$ $\mathrm{GHz}$. The center frequency $\left(f_{0}\right)=4.50 \mathrm{GHz}$, and the bandwidth $=640 \mathrm{MHz}$, as shown in Table 1 .

Table 1. The Waveguide Filter Design

\begin{tabular}{|c|c|c|c|}
\hline \multirow{2}{*}{ Variables } & \multicolumn{3}{|c|}{ Waveguide Filter Parameters } \\
\cline { 2 - 4 } & One iris & Two irises & Three irises \\
\hline Center frequency $\left(f_{0}\right)$ & $4.50 \mathrm{GHz}$ & $4.50 \mathrm{GHz}$ & $4.50 \mathrm{GHz}$ \\
\hline Bandwidth & $420 \mathrm{MHz}$ & $640 \mathrm{MHz}$ & $640 \mathrm{MHz}$ \\
\hline Width (a), mm & 64 & 64 & 64 \\
\hline High (b), mm & 32 & 32 & 32 \\
\hline Iris thickness, mm & 1.0 & 1.0 & 1.0 \\
\hline Iris width (d1), mm & 32 & 40 & 28 \\
\hline Iris width (d2), mm & - & 40 & 42 \\
\hline Iris width (d3), mm & - & - & 28 \\
\hline Length (11), mm & 48 & 48 & 48 \\
\hline Length (12), mm & 48 & 32 & 32 \\
\hline Length (13), mm & - & 48 & 32 \\
\hline Length (14), mm & - & - & 48 \\
\hline
\end{tabular}

The designed bandpass waveguide filter with three irises described in Table 1 has been tested by the CST Microwave Studio ${ }^{\circledR}$ software. The simulated results show that the center frequency $\left(f_{0}\right)=4.56 \mathrm{GHz}$, the bandwidth $=640 \mathrm{MHz}$, the lower frequency $\left(f_{I}\right)=4.24 \mathrm{GHz}$, and the upper frequency $\left(f_{2}\right)$ $=4.88 \mathrm{GHz}$. We observed that the response curve of $\mathrm{dB}(\mathrm{S} 11)$ at the stop-band frequency shows two transition ripples corresponding to the order $(\mathrm{n}=2)$. The frequency responses of the scattering parameters of $\mathrm{dB}(\mathrm{S} 11)$ and $\mathrm{dB}(\mathrm{S} 21)$ are shown in Figure 7.

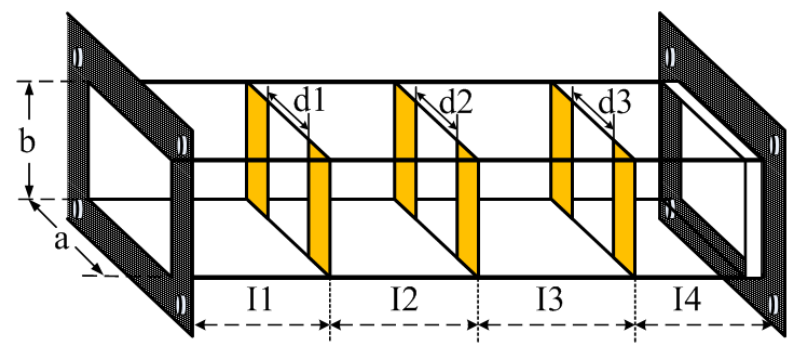

Figure 6. The bandpass waveguide iris filter with 3 irises in a cascade

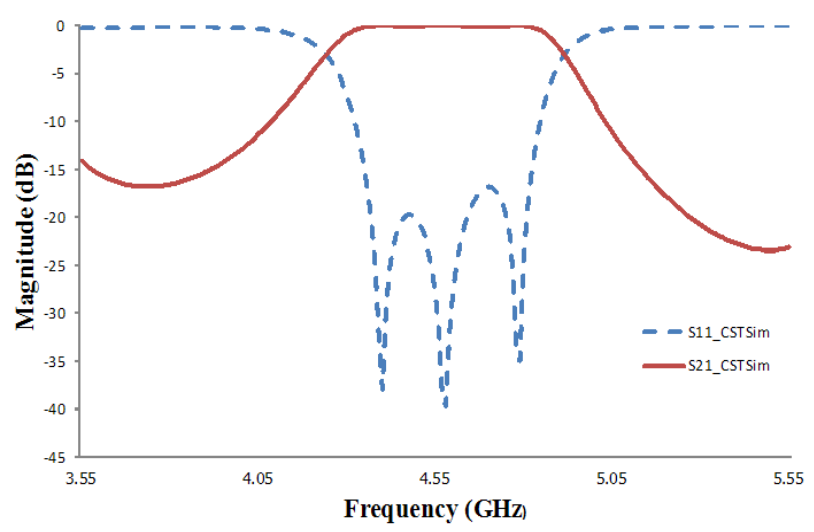

Figure 7. Simulation results by CST tools
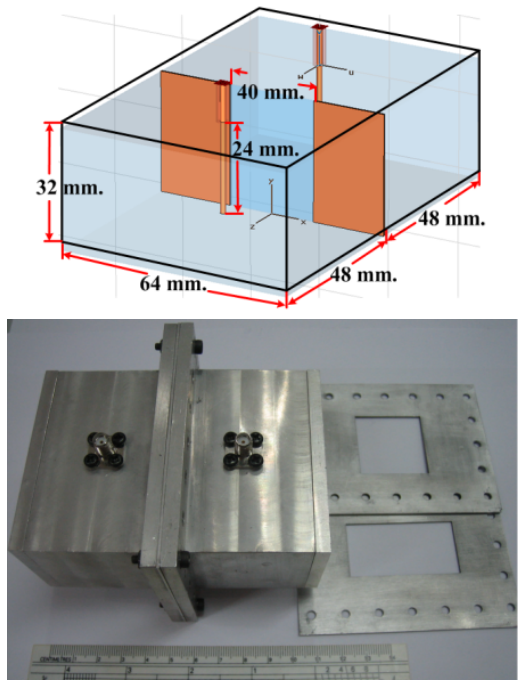

Figure 8. Simple designed waveguide filter

\subsection{The Implementation of Waveguide Filters}

The sample implementation of bandpass filters in rectangular waveguide is designed by using WFD simulation and CST Microwave Studio ${ }^{\circledR}$ software at the center frequency of approximately $4.5 \mathrm{GHz}$. The parts were made of aluminum. An example of a simple bandpass filter has been fabricated according to the configuration parameters of an inductive iris in Table 1. This iris was placed within the rectangular waveguide with a width (a) equal to $64 \mathrm{~mm}$, a height (b) equal to $32 \mathrm{~mm}$, and a length equal to $48 \mathrm{~mm}$. Port 1 and port 2 are connected by a $\lambda / 4$ vertical monopole antenna that is excited by the source of the fundamental mode $\left(T E_{10}\right)$, as depicted in Figure 8. Figure 9 shows the experimental setup of the bandpass waveguide filter measured on the N5230C network analyzer (Agilent Technologies).

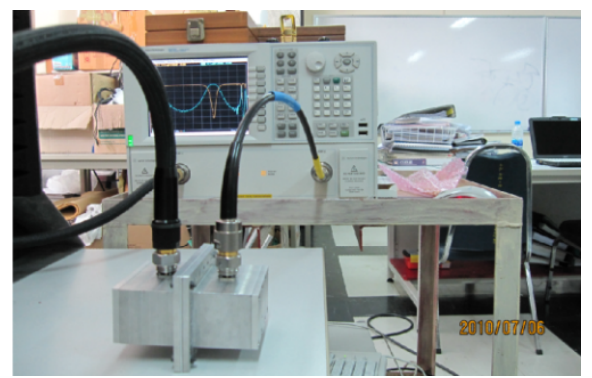

Figure 9. The experimental setup of the waveguide filter

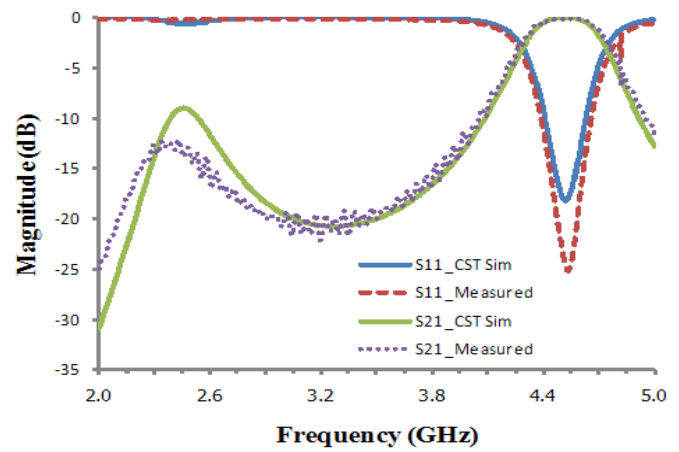

Figure 10. Comparison of $\mathrm{dB}(\mathrm{S} 11)$ and $\mathrm{dB}(\mathrm{S} 21)$ of the bandpass filter between the CST simulation and the experiment 


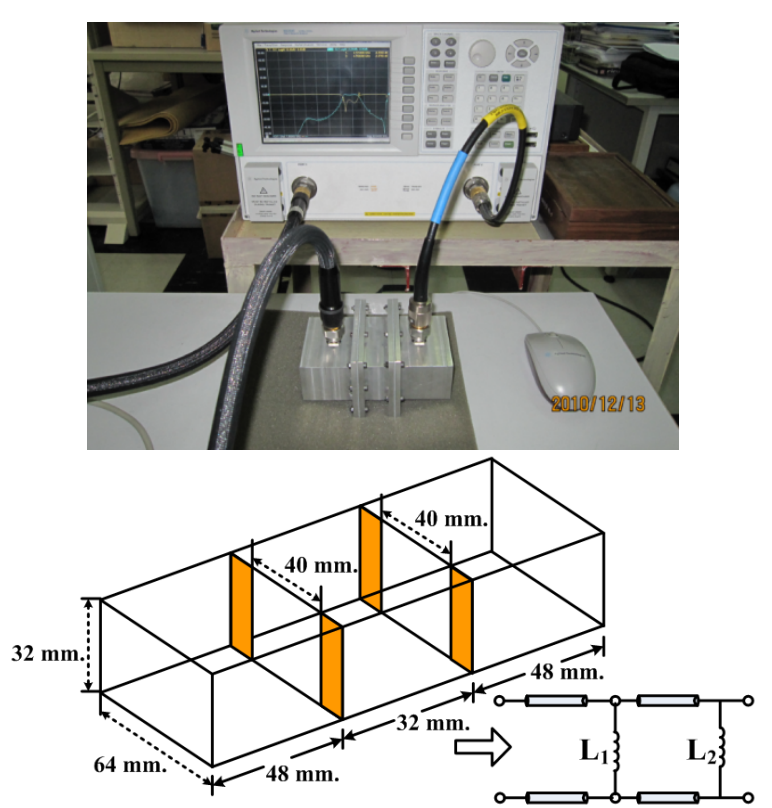

Figure 11. Configuration of two inductive irises

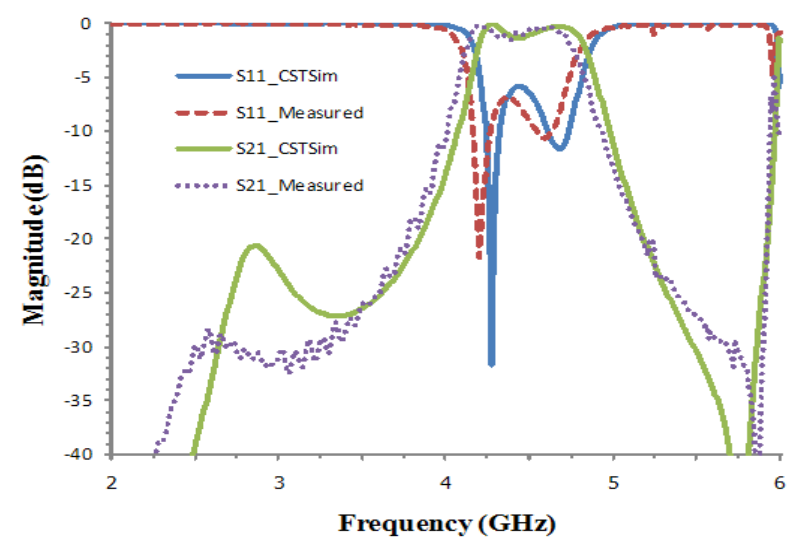

Figure 12. Simulated and measured results of a filter using two inductive irises
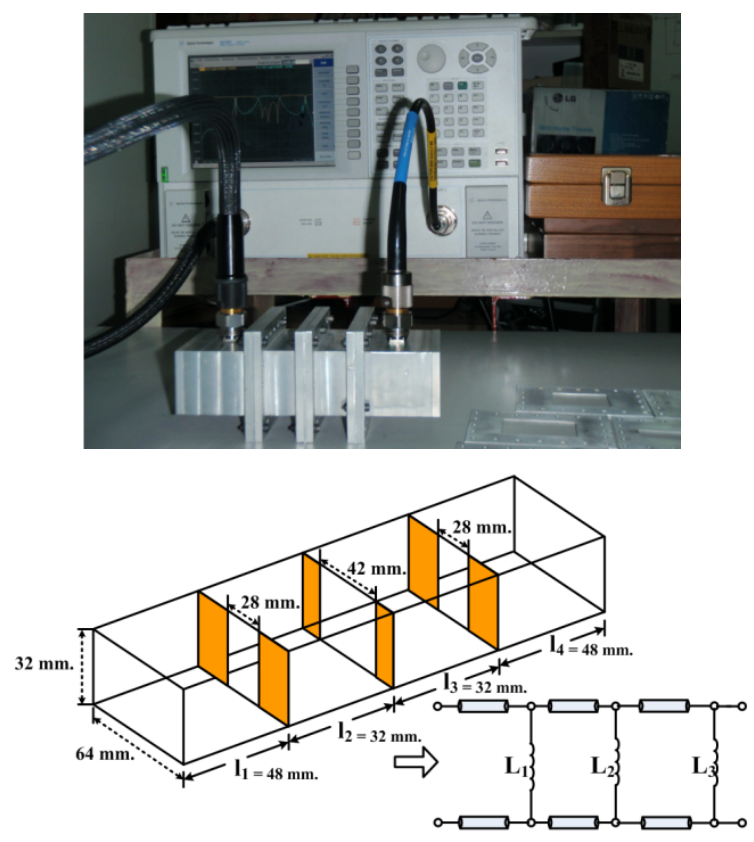

Figure 13. Configuration of three inductive irises
Figure 10 presents the frequency response of the scattering parameters of $\mathrm{dB}(\mathrm{S} 11)$ and $\mathrm{dB}(\mathrm{S} 21)$ with an iris width equal to $40 \mathrm{~mm}$. The passband frequency is 4.320 to $4.745 \mathrm{GHz}$ with a $425 \mathrm{MHz}$ bandwidth. The resonant frequency and the pass band can be controlled by varying the iris width. We can see that the measured results agree well with the CST simulated results.

Figure 11 shows the configuration of the waveguide bandpass filter with 2 inductive irises with the width of the waveguide (a) equal to $64 \mathrm{~mm}$ and the height (b) equal to 32 $\mathrm{mm}$ and the width of inductive iris circuit $1(\mathrm{~d} 1)$ and circuit 2 (d2) equal to $40 \mathrm{~mm}$. The length between circuit 1 and circuit 2 is equal to $32 \mathrm{~mm}$. We use the configuration data in Table 1 as the initial values and optimize the waveguide dimension using the CST simulation tool.

Figure 12 presents the frequency response of the scattering parameters of $\mathrm{dB}(\mathrm{S} 11)$ and $\mathrm{dB}(\mathrm{S} 21)$ to cover the frequency range from 2 to $6 \mathrm{GHz}$ for a bandpass filter with 2 inductive irises. We have observed that the passband frequency is covered in the 4.10 to $4.755 \mathrm{GHz}$ range with a $655 \mathrm{MHz}$ bandwidth. The different results are due to the implemented circuit structure and the effect of source matching impedance.

Figure 13 shows the configuration of the waveguide bandpass filter with three inductive irises. The width of the waveguide (a) is equal to $64 \mathrm{~mm}$ and the height (b) is equal to $32 \mathrm{~mm}$. The circuit parameters are the width of circuit 1 (d1) and circuit $3(\mathrm{~d} 3)$, which are equal to $28 \mathrm{~mm}$, and the width of circuit 2 (d2), which is equal to $42 \mathrm{~mm}$. The length of each iris is equal to $32 \mathrm{~mm}$ and $48 \mathrm{~mm}$. We use the configuration data in Table 1 as the initial values and optimize the waveguide dimension by using CST simulation. The frequency response is presented in Figure 14.

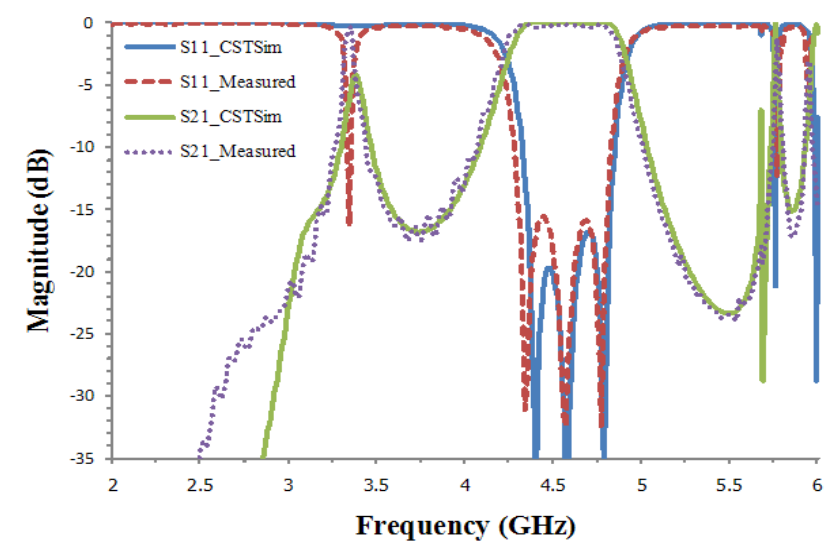

Figure 14. Simulated and measured results of a filter with 3 inductive irises

Figure 14 presents the frequency response of the scattering parameters of $\mathrm{dB}(\mathrm{S} 11)$ and $\mathrm{dB}(\mathrm{S} 21)$ to cover the frequency range from 2 to $6 \mathrm{GHz}$ using three inductive irises. The pass-band frequency is $4.240-4.885 \mathrm{GHz}$ with a $645 \mathrm{MHz}$ bandwidth. We observed that the return losses having a -17 $\mathrm{dB}$ maximum at the stop-band frequency shows two transition ripples corresponding to the order $(n=2)$. The measured results agree with the CST simulation. The error in the op- 
erating frequency range is equal to approximately $\pm 4.5 \%$ because of the effect of the usable dipole antenna and the thinness of the aluminum material. The fabricated and measured conditions also contributed to the error.

\section{Conclusions}

This paper presents the WFD simulation, design and implementation of bandpass filters in rectangular waveguides. The WFD (Waveguide Filter Design) simulation based on an iterative method and wave propagation has been proposed for an inductive iris analysis. The capability of the WFD simulation has been validated. It appears to be an efficient alternative to the CST simulation. The performance of the implemented waveguide filters confirms the suitability of the developed WFD and CST simulation because the error is less than $\pm 5 \%$ compared with the designed filters.

The WFD simulation allows us to reduce the design problem to determinate a bandpass filter structure in the rectangular waveguide with usable frequency range. An interesting aspect of WFD simulation is its application to the design and analysis of different microwave planar circuits. In addition, this simulation can be used as a teaching aid in the microwave engineering education.

\section{ACKNOWLEDGEMENTS}

The authors would like to thank the Faculty of Technical Education, Rajamangala University of Technology Thanyaburi for providing the network analyzer N5230C model of Agilent Technologies and CST Microwave Studio ${ }^{\circledR}$ for measurement and design.

\section{REFERENCES}

[1] T. Hiraoka, C. P. Chen, T. Anada, J. P. Hsu, Z. W. Ma, and C. C. Christopoulos, "Electric Field Distributions in Microwave Planar Circuits by Ssmall Ccoaxial Probe and Comparison with FDTD Method," in Microwave Conference, 2005 European, pp. 269-272, 2005.

[2] A. Mediavilla, A. Tazon, J. A. Pereda, M. Lazaro, I. Santamaria, and C. Pantaleon, "Neuronal Aarchitecture for Wave Guide Inductive Iris Bandpass Filter Optimization," in Neural Networks, IJCNN 2000, Proceedings of the IEEE-
INNS-ENNS International Joint Conference, pp. 395-399, vol.4, 2000.

[3] J. W. Bandler, A. S. Mohamed, and M. H. Bakr, "TLM-based Modeling and Design Exploiting Space Mapping," Microwave Theory and Techniques, IEEE Transactions, vol. 53, pp. 2801-2811, 2005.

[4] S. Seunghyun, K. Hyeong-Seok, J. Hyun-Kyo, J. Braunstein, and M. Un-Chul, "Frequency Domain Analysis of Microstrip Filters and Antennae using an Adaptive Frequency Sampling Moment Method," Magnetics, IEEE Transactions, vol. 42, pp. 607-610, 2006.

[5] V. K. Chaudhary, P. Verma, and U. Balaji, "Field Theory Based CAD of Inductive Iris Waveguide Filter," APMC 2001. Asia Pacific Microwave Conference, pp. 318-321 vol.1. 2001.

[6] M. Ohira, H. Deguchi, M. Tsuji, and H. Shigesawa, "Novel Waveguide Filters with Multiple Attenuation Poles using Dual-Behavior Resonance of Frequency-Selective Surfaces", Microwave Theory and Techniques, IEEE Transactions, vol. 53, pp. 3320-3326, 2005.

[7] V. E. Boria and B. Gimeno, "Waveguide filters for satellites", Microwave Magazine, IEEE, vol. 8, pp. 60-70, 2007.

[8] A. Sallier, J. Bornemann, and W. J. R. Hoefer, "Field-Based Waveguide Filter Synthesis in the Time Domain", AEU International Journal of Electronics and Communications, vol. 57, pp. 119-127, 2003.

[9] I. Hirsh, M. Horowitz and A. Rosenthal, "Design of Planar Waveguides with Prescribed Mode-Profile using Inverse Scattering Theory", Quantum Electronics, IEEE Journal of, vol.45, no.9, pp. 1133-1141, 2009.

[10] Y. P. Zhang, H. Guorui, Z. Wenmei and J. H. Sheng, "Electromagnetic Mode Theory of Periodically-Loaded Oversized Imperfect Waveguide and its Application to the Propagation of Radio Waves in Long Wall Coal Mining Face Tunnels", Antennas and Propagation, IEEE Transactions, vol.58, no.5, pp. 1816-1822, 2010.

[11] P. Soto, Tari, x, E. n, V. E. Boria, C. Vicente, J. Gil, and B. Gimeno, "Accurate Synthesis and Design of Wideband and Inhomogeneous Inductive Waveguide Filters", Microwave Theory and Techniques, IEEE Transactions, vol. 58, pp. 2220-2230, 2010.

[12] S. Choocadee and S. Akatimagool, "Development of Efficiency EM Simulation Tool for Capacitive and Inductive Obstacle Analysis", in Electrical Engineering/Electronics Computer Telecommunications and Information Technology, International Conference, pp. 1154-1158, 2010. 